\title{
Applications of Nambu Mechanics to Systems of Hydrodynamical Type II
}

\author{
Partha GUHA
}

S.N. Bose National Centre for Basic Sciences, JD Block, Sector-3, Salt Lake

Calcutta-700098, INDIA

E-mail: partha@bose.res.in

Received September 12, 2003; Accepted January 15, 2004

\begin{abstract}
In this paper we further investigate some applications of Nambu mechanics in hydrodynamical systems. Using the Euler equations for a rotating rigid body Névir and Blender [J. Phys. A 26 (1993), L1189-L1193] had demonstrated the connection between Nambu mechanics and noncanonical Hamiltonian mechanics. Nambu mechanics is extended to incompressible ideal hydrodynamical fields using energy and helicity in three dimensional (enstrophy in two dimensional). In this paper we discuss the Lax representation of systems of Névir-Blender type. We also formulate the three dimensional Euler equations of incompressible fluid in terms of Nambu-Poisson geometry. We discuss their Lax representation. We also briefly discuss the Lax representation of ideal incompressible magnetohydrodynamics equations.
\end{abstract}

\section{Introduction}

The configuration space of an incompressible fluid is the group of volume preserving diffeomorphisms of the three-dimensional region in $\mathbf{R}^{3}$ containing the fluid.

Arnold [1] showed in 1966 that, if $u(x, t)$ is a time-dependent divergence-free vector field on a compact Riemannian n-manifold $M$ and if $\eta(x, t)$ is the volume-preserving flow, then $u$ satisfies the Euler equation

$$
\frac{\partial u}{\partial t}+\nabla_{u} u=-\operatorname{grad} P \quad \mathrm{P}=\text { Pressure }
$$

if and only if the curve $t \longmapsto \eta(\cdot, t)$ is an $L^{2}$ geodesic in $\mathcal{D}_{\mu}(M)$, the group of $C^{\infty}$ volume preserving diffeomorphism on $M$.

In a celebrated paper Ebin and Marsden [2] developed the analytic geometrical side of Arnold's paper. They showed that the spray of the Euler equation is smooth. They also proved that on a manifold without boundary the solutions of the Navier-Stokes equation converge to those of the Euler equations when the viscosity tends to zero.

In spite of the powerful analytical techniques in the study of fluid dynamics, it has been accepted [3] that the three dimensional Euler equation requires more geometrical tools to unveil this subject. 
In this paper we explore the 3D Euler equation from the Nambu-Poisson geometric point of view. In a remarkable paper Nambu [4] initiated the study of multi-Hamiltonian systems. Nambu's paper was followed by a number of papers $[5,6,7]$ which essentially carry the idea from the physical point of view by showing that the classical part of it could be cast into the frame work of classical mechanics with Dirac constraint.

Recently this research was revitalized by Takhtajan [8]. He presented a beautiful theory, revealing deep and nontrivial connection with algebra and differential geometry. The Nambu-Poisson structure leads to interesting mathematical developments, several authors have studied Lie-algebraic structures $[9,10,11,12,13,14,15,16,17,18]$ on one hand and generalized Hamiltonian dynamics $[19,20]$ on other hand.

R. Chatterjee [21] had shown that several Hamiltonian systems possessing dynamical or hidden symmetries can be realized within the framework of Nambu mechanics. Among the notable examples are the $S U(n)$ isotropic harmonic oscillator and the $S O(4)$ Kepler problem. Most recently Gonera and Nutku [22] showed that the Nambu structure can be extracted also from the rational Calogero-Moser system [23].

Our project is to explore different applications of Nambu mechanics to various hydrodynamical systems. In our earlier paper [24] we showed that the reduced Dubrovin-Novikov [25] hydrodynamic type models are integrable Nambu mechanical systems admitting Lax triples. In this paper we study the celebrated 3D Euler equation and the ideal incompressible magnetohydrodynamic equation.

\section{Preliminaries}

A generalization of classical Hamiltonian mechanics was proposed by Y. Nambu [4]; he replaced a pair of variables in the Hamiltonian formulation by an $n$-tuple of variables, where $n \geq 3$, and the Poisson bracket by an $n$-ary operation, the Nambu bracket. For $n=3$, Nambu apparently introduced his bracket in order to develop a toy model for quarks considered as triples.

The modern concept of a Nambu-Poisson structure was proposed by Takhtajan in 1994 [8] in order to find an axiomatic formulation for the $n$-bracket operation. Let $M$ denote a smooth $n$-dimensional manifold and $C^{\infty}(M)$ the algebra of infinitely differentiable real valued functions on $M$.

Definition 2.1. A manifold $M$ is called a Nambu-Poisson manifold if there exists a $\mathbb{R}$ multi-linear map

$$
\{, \ldots,\}:\left[C^{\infty}(M)\right]^{\otimes n} \rightarrow C^{\infty}(M) .
$$

This is called a Nambu-Poisson bracket of order $n \forall f_{1}, f_{2}, \ldots, f_{2 n-1} \in C^{\infty}(M)$. This bracket satisfies

1. $\left\{f_{1}, \ldots, f_{n}\right\}=(-1)^{\epsilon(\sigma)}\left\{f_{\sigma(1)}, \ldots, f_{\sigma(n)}\right\}$,

2. $\left\{f_{1} f_{2}, f_{3}, \ldots, f_{n+1}\right\}=f_{1}\left\{f_{2}, f_{3}, \ldots, f_{n+1}\right\}+\left\{f_{1}, f_{3}, \ldots, f_{n+1}\right\} f_{2}$,

3. It satisfies the fundamental identity

$$
\begin{gathered}
\left\{\left\{f_{1}, \ldots, f_{n-1}, f_{n}\right\}, f_{n+1}, \ldots, f_{2 n-1}\right\}+\left\{f_{n},\left\{f_{1}, \ldots, f_{n-1}, f_{n+1}\right\}, f_{n+2}, \ldots, f_{2 n-1}\right\}(2.2) \\
+\cdots+\left\{f_{n}, \ldots, f_{2 n-2},\left\{f_{1}, \ldots, f_{n-1}, f_{2 n-1}\right\}\right\}=\left\{f_{1}, \ldots, f_{n-1},\left\{f_{n}, \ldots, f_{2 n-1}\right\}\right\},
\end{gathered}
$$


where $\sigma \in S_{n}$ - the symmetric group of $n$ elements - and $\epsilon(\sigma)$ is its parity.

This fundamental identity is called the Takhtajan identity.

Let $\{\bullet, \bullet, \cdots, \bullet\}: C^{\infty}(M) \times C^{\infty}(M) \cdots \times C^{\infty}(M) \longrightarrow C^{\infty}(M)$ be a multi derivation satisfies Takhtajan identity iff

1. $\{\bullet, \bullet, \cdots, \bullet\}$ satisfies the Takhtajan identity for generators.

2. $\{\bullet, \bullet, \cdots, \bullet\}$ satisfies quadratic identities

$$
\begin{array}{r}
\sum_{k=1}^{n}\left\{\phi, f_{1}, \cdots, f_{n-2}, f_{n+k-1}\right\}\left\{\phi^{\prime}, f_{n}, \cdots, \widehat{f_{n+k-1}}, \cdots, f_{2 n-1}\right\} \\
+\left\{\phi^{\prime}, f_{1}, \cdots, f_{n-2}, f_{n+k-1}\right\}\left\{\phi, f_{n+1}, \cdots, \widehat{f_{n+k-1}}, \cdots, f_{2 n-1}\right\}=0 .
\end{array}
$$

Definition 2.2. Let $M$ be a smooth finite $n$-dimensional manifold with algebra of functions $C^{\infty}(M)$. The Lie algebra of vector fields $\chi(M)$

$$
X:\left[C^{\infty}(M)\right]^{\otimes(n-1)} \longrightarrow \chi(M)
$$

$\forall f_{1}, f_{2}, \ldots, f_{n-1} \in C^{\infty}(M)$

$$
\left(f_{1}, \ldots, f_{n-1}\right) \longmapsto X_{f_{1}, \ldots, f_{n-1}} .
$$

such that the bracket defined by

$$
\left\{f, f_{1}, \ldots, f_{n-1}\right\}:=X_{f_{1} \ldots f_{n-1}} f
$$

is skew symmetric in all arguments and is invariant under any Hamiltonian vector fields $X=X_{f_{1} \ldots f_{n-1}}$, i.e.

$$
X\left\{f_{n}, \ldots, f_{2 n-1}\right\}=\left\{X f_{n}, \ldots, f_{2 n-1}\right\}+\cdots+\left\{f_{n}, \cdots, X f_{2 n-1}\right\} .
$$

It is known that the Nambu dynamics on a Nambu-Poisson phase space involves $n-1$ so-called Nambu-Hamiltonians $H_{1}, \ldots, H_{n-1} \in C^{\infty}(M)$ and is governed by the following equations of motion

$$
\frac{d f}{d t}=\left\{f, H_{1}, \ldots, H_{n-1}\right\}, \forall f \in C^{\infty}(M) .
$$

A solution to the Nambu-Hamilton equations of motion produces an evolution operator, $U_{t}$, which by virtue of the fundamental identity preserves the Nambu bracket structure on $C^{\infty}(M)$.

Definition 2.3. $f \in C^{\infty}(M)$ is a first integral of $X_{H_{1}, \cdots H_{n-1}}$ if and only if

$$
\left\{f, H_{1}, H_{2}, \cdots, H_{n-1}\right\}=0 .
$$

It is known that the Nambu-Poisson what includes all the subordinate Poisson structures. Thus the Poisson structure on $\mathbb{R}^{3}$ can be written as

$$
\left\{x_{i}, x_{j}\right\}^{F}:=\left\{x_{i}, x_{j}, F\right\} \quad x_{i}=x, y, z .
$$


Lemma 2.4. The bracket $\{f, g\}^{H}=\{f, g, H\}$ satisfies Jacobi identity.

Proof: The Jacobi identity of $f, g$ and $h$ is given by

$$
\begin{gathered}
\left\{\{f, g\}^{H}, h\right\}^{H}+\left\{\{g, h\}^{H}, f\right\}^{H}+\left\{\{h, f\}^{H}, g\right\}^{H} \\
=\{\{f, g, H\}, h, H\}+\{\{g, h, H\}, f, H\}+\{\{h, f, H\}, g, H\} \\
=0 \quad \text { follows from Takhtajan's identity }(3)
\end{gathered}
$$

\subsection{Névir-Blender formulation of hydrodynamics}

The first attempt to make a connection between Nambu mechanics and the Euler equation of incompressible fluid was proposed by Névir and Blender [26]. They showed that the Nambu mechanics can be incorporated into an incompressible ideal hydrodynamical fields using energy and helicity in three dimensional. The result is a generalization of the noncanonical Hamiltonian formultaion proposed by Olver [27], in which the Poisson bracket is replaced by multilinear antisymmetric bracket.

The noncanonical Hamiltonian theory of perfect fluid dynamics for the Eulerian variables is characterized by the existence of Casimir functions like helicity and enstrophy in three and two-dimensional spaces respectively. Motivated by Nambu's approach, Névir and Blender proposed an extension of his theory to hydrodynamical fields in which these Casimirs act as Hamiltonians for Nambu mechanics.

Incompressible inviscid fluid dynamics in 3D is governed by the vorticity equation

$$
\partial_{t} \Omega+(u \cdot \nabla) \Omega-(\Omega \cdot \nabla) u=0,
$$

where $u=\left(u_{1}, u_{2}, u_{3}\right)$ is the velocity, $\Omega=\left(\Omega_{1}, \Omega_{2}, \Omega_{3}\right)$ is the vorticity, $\nabla=\left(\partial_{x}, \partial_{y}, \partial_{z}\right)$, $\Omega=\nabla \times u$, and $\nabla \cdot u=0$.

The total energy and

$$
H=\frac{1}{2} \int d^{3} x u^{2}=-\frac{1}{2} \int d^{3} x \Omega \cdot A
$$

and the total helicity

$$
h=\frac{1}{2} \int d^{3} x \Omega \cdot u
$$

are conserved (it is assumed that $u$ vanishes at infinity) and $A$ is a vector potential such that $u=-\nabla \times A$ and $\nabla \cdot A=0$.

The identity $u \cdot \nabla u=\frac{1}{2} \nabla|u|^{2}-u \times(\nabla \times u)$ can be used to bring the Euler equation (1) into the form

$$
\frac{\partial u}{\partial t}-u \times(\nabla \times u)=-\nabla\left(\frac{1}{\rho} P+\frac{1}{2} u^{2}\right) .
$$

Thus in terms of vorticity the Euler equation is

$$
\frac{\partial \Omega}{\partial t}-\nabla \times(u \times \Omega)=0,
$$

where $P$ denotes pressure. 
The noncanonical form of the vorticity equation is

$$
\frac{\partial \Omega}{\partial t}=J(\Omega) \frac{\delta H}{\delta \Omega}
$$

with the antisymmetric operator

$$
J(\Omega)=-\nabla \times(\Omega \times \nabla \times(.)) .
$$

The derivative of the energy with respect to the vorticity is given by $\frac{\delta H}{\delta \Omega}=-A$.

Let $F=F(\Omega)$ be an arbitrary functional. Névir and Blender proposed an evolution equation for $F(\Omega)$ according to

$$
\frac{\partial F}{\partial t}:=-\int d^{3} x\left(\nabla \times \frac{\delta F}{\delta \Omega}\right) \times\left(\nabla \times \frac{\delta h}{\delta \Omega}\right) \cdot\left(\nabla \times \frac{\delta H}{\delta \Omega}\right)=-\{F, h, H\} .
$$

Proposition 2.5. The Lax triple of the Névir-Blender equation

$$
\frac{\partial F}{\partial t}+\{F, h, H\}=0, \text { where }\{F, h, H\}=\frac{\partial(F, h, H)}{\partial(x, y, z)},
$$

is given by

1. $\left\{F, \phi_{1}, \phi_{2}\right\}=\lambda \phi_{2}$,

2. $\partial \phi_{1}=\left\{h, H, \phi_{1}\right\}$,

3. $\partial \phi_{2}=\left\{h, H, \phi_{2}\right\}$.

Sketch of the Proof: We differentiate the first equation with respect to time $t$. We obtain

$$
\begin{gathered}
\left\{F_{t}, \phi_{1}, \phi_{2}\right\}+\left\{F, \phi_{1 t}, \phi_{2}\right\}+\left\{F, \phi_{1}, \phi_{2 t}\right\}=\left\{h, H,\left\{F, \phi_{1}, \phi_{2}\right\}\right\} \\
\left\{F_{t}, \phi_{1}, \phi_{2}\right\}+\left\{F,\left\{h, H, \phi_{1}\right\}, \phi_{2}\right\}+\left\{F, \phi_{1},\left\{h, H, \phi_{2}\right\}\right\}=\left\{h, H,\left\{F, \phi_{1}, \phi_{2}\right\}\right\} .
\end{gathered}
$$

By using the Takhtajan identity

$$
\begin{array}{r}
\left\{\left\{f_{1}, f_{2}, f_{3}\right\}, f_{4}, f_{5}\right\}+\left\{f_{3},\left\{f_{1}, f_{2}, f_{4}\right\}, f_{5}\right\} \\
+\left\{f_{3}, f_{4},\left\{f_{1}, f_{2}, f_{5}\right\}\right\} \\
=\left\{f_{1}, f_{2},\left\{f_{3}, f_{4}, f_{5}\right\}\right\} .
\end{array}
$$

we obtain our desired result.

Similarly Névir and Blender showed that the vorticity equation of incompressible fluid flow in two dimensions can be recasted to

$$
\frac{\partial \Omega}{\partial t}=-\mathcal{J}\left(\frac{\delta \mathcal{E}}{\delta \Omega}, \frac{\delta H}{\delta \Omega}\right)
$$

where $\mathcal{E}=\frac{1}{2} \int d^{2} x \Omega^{2}$ is known as enstrophy and $\mathcal{J}$ is the Jacobi operator, $\mathcal{J}(p, q)=$ $\partial_{x} p \partial_{y} q-\partial_{y} p \partial_{x} q$.

REMARK: The Lax pair of the 2D Euler equation was first given by Friedlander and Vishik $[28,29]$, in terms of Lagrangian coordinates, and most recently by $\mathrm{Li}[30]$ in terms of 
Eulerian coordinates. If we go to the two-dimensional case our Lax representation exactly coincides with the Lax pair of the 2D Euler equation

$$
L \phi=\lambda \phi, \quad \partial_{x} \phi+A \phi=0,
$$

where $L \phi=\{\Omega, \phi\}, A \phi+\{\chi, \phi\}=0$, and the compatibility condition yields

$$
\partial_{x} L+[A, L]=\partial_{x} \Omega+\{\Omega, \chi\}=0 .
$$

It was shown [26] that time evolution of an arbitrary functional $F=F[\Omega]$ in $2 \mathrm{D}$ is given by

$$
\frac{\partial F}{\partial t}=-\int d^{2} x \frac{\delta F}{\delta \Omega} \mathcal{J}\left(\frac{\delta \mathcal{E}}{\delta \Omega}, \frac{\delta H}{\delta \Omega}\right)=\{F, \mathcal{E}, H\}_{2 D} .
$$

It is clear from lemma $(2.4)$ that $\{F, \mathcal{E}, H\}_{2 D}$ satisfies Jacobi's identity.

\section{Reformulation of 3D Euler equation}

We consider the vorticity equation of the Euler equation of incompressible fluid. We introduce the first pair of Clebsch variables, $\alpha$ and $\beta$, such that it satisfies

$$
\mathbf{u}=\alpha \nabla \beta+\nabla \gamma
$$

Hence $\Omega=\nabla \times \mathbf{u}$ as

$$
\Omega=\nabla \alpha \times \nabla \beta \text {. }
$$

It is easy to show that $\alpha$ and $\beta$ satisfy the continuity or transport equations, that is

$$
\begin{aligned}
& \frac{\partial \alpha}{\partial t}+\mathbf{u} \cdot \nabla \alpha=0 \\
& \frac{\partial \beta}{\partial t}+\mathbf{u} \cdot \nabla \beta=0 .
\end{aligned}
$$

At this stage we introduce another set of variables, $\chi_{1}$ and $\chi_{2}$, such that

$$
\mathbf{u}=\nabla \chi_{1} \times \nabla \chi_{2}
$$

The newly introduced pair, $\left(\chi_{1}, \chi_{2}\right)$, is not totally independent of $(\alpha, \beta)$. Since

$$
\nabla \times\left(\nabla \chi_{1} \times \nabla \chi_{2}\right)=(\nabla \alpha \times \nabla \beta)
$$

We rewrite the the vorticity equation as

$$
\Omega_{t} \cdot \nabla \phi+u \cdot \nabla(\Omega \cdot \nabla \phi)-\Omega \cdot \nabla(u \cdot \nabla \phi)=0,
$$

Lemma 3.1. The following system of equations is two equivalent expressions of the $3 \mathrm{D}$ Euler equation:

$$
\Omega_{t} \cdot \nabla \phi+u \cdot \nabla(\Omega \cdot \nabla \phi)-\Omega \cdot \nabla(u \cdot \nabla \phi)=0
$$

and

$$
\left(\nabla \alpha_{t} \times \nabla \beta\right) \cdot \nabla \phi+\left(\nabla \alpha \times \nabla \beta_{t}\right) \cdot \nabla \phi+\left\{\chi_{1}, \chi_{2},\{\alpha, \beta\}, \phi\right\}-\left\{\alpha, \beta,\left\{\chi_{1}, \chi_{2}\right\}, \phi\right\}=0
$$

or

$$
\left\{\alpha_{t}, \beta, \phi\right\}+\left\{\alpha, \beta_{t}, \phi\right\}+\left\{\chi_{1}, \chi_{2},\{\alpha, \beta\}, \phi\right\}-\left\{\alpha, \beta,\left\{\chi_{1}, \chi_{2}\right\}, \phi\right\}=0 .
$$


Sketch of Proof: We have used the identity $(\nabla A \times \nabla B) \cdot \nabla C=\{A, B, C\}_{3 D}$.

By these new transformations the continuity equations become

$$
\begin{aligned}
& \frac{\partial \alpha}{\partial t}+\left\{\chi_{1}, \chi_{2}, \alpha\right\}=0 \\
& \frac{\partial \beta}{\partial t}+\left\{\chi_{1}, \chi_{2}, \beta\right\}=0 .
\end{aligned}
$$

Lemma 3.2. The Lax representation of the 3D Euler equation is given by

$$
\begin{aligned}
& \{\alpha, \beta, \phi\}=\lambda \phi, \\
& \frac{\partial \phi}{\partial t}+\left\{\chi_{1}, \chi_{2}, \phi\right\}=0 .
\end{aligned}
$$

Proof: It is easy to show that the compatibility condition

$$
\left\{\alpha_{t}, \beta, \phi\right\}+\left\{\alpha, \beta_{t}, \phi\right\}+\left\{\alpha, \beta,\left\{\chi_{1}, \chi_{2}, \phi\right\}\right\}=\left\{\chi_{1}, \chi_{2}\{\alpha, \beta, \phi\}\right\}
$$

exactly coincides with equation (28).

To prove the above identity one requires transport equations and the Takhtajan identity

$$
\begin{array}{r}
\left\{\left\{f_{1}, f_{2}, f_{3}\right\}, f_{4}, f_{5}\right\}+\left\{f_{3},\left\{f_{1}, f_{2}, f_{4}\right\}, f_{5}\right\} \\
+\left\{f_{3}, f_{4},\left\{f_{1}, f_{2}, f_{5}\right\}\right\} \\
=\left\{f_{1}, f_{2},\left\{f_{3}, f_{4}, f_{5}\right\}\right\} .
\end{array}
$$

Thus we obtain our desired result.

REMARK: The above Lax formulation is equivalent to some other Lax pair of the 3D Euler equation of incompressible fluid flow

$$
(\Omega \cdot \nabla) \phi=\lambda \phi \quad \partial_{t} \phi+(\mathbf{u} \cdot \nabla) \phi=0 .
$$

It is easy to show that the compatibility condition yields $\Omega_{t}+(\mathbf{u} \cdot \nabla) \Omega-(\Omega \cdot \nabla) \mathbf{u}=0$.

Proposition 3.3. The 3D Euler equation is described by the Lax triple

$$
\begin{aligned}
& \frac{\partial \phi}{\partial t}+\left\{\chi_{1}, \chi_{2}, \phi\right\}=0 \\
& \frac{\partial \alpha}{\partial t}+\left\{\chi_{1}, \chi_{2}, \alpha\right\}=0 \\
& \frac{\partial \beta}{\partial t}+\left\{\chi_{1}, \chi_{2}, \beta\right\}=0
\end{aligned}
$$

and $\{\alpha, \beta, \phi\}=\lambda \phi$. 


\section{Applications to Ideal incompressible MHD}

The purpose of this section is to show that the equation of ideal incompressible magnetohydrodynamics (MHD) has a similar Nambu structure. The equations of ideal incompressible MHD coupled with an inviscid fluid to a magnetic field $B$ are

$$
\begin{aligned}
& \frac{\partial \mathbf{u}}{\partial t}+(\mathbf{u} \cdot \nabla) \mathbf{u}=B \cdot \nabla B-\nabla p \\
& \frac{\partial B}{\partial t}=B \cdot \nabla \mathbf{u},
\end{aligned}
$$

together with

$$
\operatorname{div} \mathbf{u}=0 \quad \text { and } \operatorname{div} B=0 .
$$

These equations can be reformulated using Elsasser variables [31]

$$
\mathbf{v}^{ \pm}=\mathbf{u} \pm B
$$

and the "vorticity" vector $\Omega^{ \pm}=$curl $\mathbf{u}$

$$
\frac{D^{ \pm} \Omega^{\mp}}{D t}=\left(\Omega^{\mp} \cdot\right) \mathbf{v}^{ \pm}
$$

where

and the magnetic field $B$ satisfies

$$
\frac{D^{ \pm}}{D t}=\frac{\partial}{\partial t}+\mathbf{v}^{ \pm} \cdot \nabla
$$

$$
\frac{D^{ \pm} B}{D t}=(B \cdot \nabla) \mathbf{v}^{ \pm}
$$

together with $\operatorname{div} \mathbf{v}^{ \pm}=0$.

It is clear from our discussions that the all these equations (33-34) satisfy the same type Nambu equations as the 3D-Euler equations. The Lax representation of all of these equation can be given easily by mimicing the previous section.

\section{Conclusion and Outlook}

In this paper we have studied 3D Euler equation of incompressible fluid using Nambu mechanics. We have extended the results of Névir and Blender who had extended Nambu mechanics to incompressible hydrodynamics.

We have proposed a Lax representation of this equation. We also outlined the Lax representation of ideal incompressible MHD equation. The Lax formalism can be easily extended to noncommutative framework. Noncommutative geometry has recently been involved in a noncommutaive gauge theory related to strings and has been stimulated by different works on field theories defined over noncommutative spaces. Noncommutative gauge theories are naively realized from ordinary commutative theories just by replacing Nambu bracket of the fields with Moyal-star bracket. Since the $\star$ product respects the skew symmetry condition, the Lax representation ( $\star$ product version ) given in this paper will also work for noncommutative case.

Another possible direction one can extend this work is to generalize the present construction to super-3D Euler flow. It involves super Nambu bracket. This is an interesting topic. 


\section{Acknowledgement}

It is a pleasure to thank Professors Jerry Marsden, Stephen Montgomery-Smith, Pol Vanhaecke and Charles Li for stimulating discussions. The author thanks Professor Stephen Montgomery-Smith for his valuable information about the 3D Lax pair. He is also grateful to the anonymous referee for many valuable suggestions. He thanks the staff of Department of Mathematics, University of Colorado at Colorado Springs, for their kind hospitality during his visit, when the essential part of this work was done.

\section{References}

[1] Arnold V I, Sur la géométrie differentielle des groupes de Lie de dimenson infinie et ses applications à l'hydrodynamique des fluids parfaits, Ann. Inst. Fourier, Grenoble 16 (1966), $319-361$.

[2] Ebin D. and Marsden J E, Groups of diffeomorphisms and the motion of an incompressible fluid, Ann. Math. 92 (1970), 102-163.

[3] Marsden J E, Private Communications.

[4] Nambu Y, Generalized Hamiltonian Mechanics, Phys. Rev. D 7 (1973), 2405.

[5] Bayen F and Flato M, Remarks concerning Nambu's generalized mechanics, Phys. Rev. D 11 (1975), 3049.

[6] Cohen I and Kálnay A J, On Nambu's generalized Hamiltonian mechanics, Internat. J. Theoret. Phys. 12 (1975), 61-67.

[7] Mukunda N and Sudarshan E C G, Relations between Nambu and Hamiltonian mechanics, Phys. Rev. D 13 (1976), 2846.

[8] Takhtajan L, On Foundation of the Generalized Nambu Mechanics, Comm. Math. Phys. 160 (1994), 295.

[9] Alekseevsky D and Guha P, On decomposability of Nambu-Poisson tensor, Acta Math. Univ. Comenian. (N.S.) 65 (1996), 1.

[10] Azcárraga J A and Prez Bueno J C, Higher-order simple Lie algebras, Comm. Math. Phys. 184 (1997), 669.

[11] Azcärraga J A, Perelomov A M and Prez Bueno J C, The Schouten-Nijenhuis bracket, cohomology and generalized Poisson structures, J. Phys. A 29 (1996) no. 24, 7993.

[12] Grabowski J and Marmo G, Remarks on Nambu-Poisson and Nambu-Jacobi brackets, J. Phys. A 32 (1999) no. 23, 4239.

[13] Grabowski J and Marmo G, On Filippov algebroids and multiplicative Nambu-Poisson structures, Differential Geom. Appl. (2000) 12 no. 1, 35.

[14] Marmo G, Vilasi G and Vinogradov A M, The local structure of $n$-Poisson and $n$-Jacobi manifolds, J. Geom. Phys. 25 (1998), 141.

[15] Michor P and Vaisman I, A note on $n$-ary Poisson brackets, The Proceedings of the 19th Winter School "Geometry and Physics" (Srn, 1999). Rend. Circ. Mat. Palermo (2) Suppl. No. 63 (2000) 165. 
[16] Michor P and Vinogradov A M, n-ary Lie and associative algebras. Geometrical structures for physical theories, II (Vietri, 1996), Rend. Sem. Mat. Univ. Politec. Torino 54 (1996) no. 4,373 .

[17] Vaisman I, A Survey on Nambu-Poisson Brackets, Acta Math. Univ. Comenian. (N.S.) 68 (1999), 213.

[18] Vaisman I, Nambu-Lie groups, J. Lie Theory 10 (2000) no. 1, 181.

[19] Guha P, Volume Preserving Multidimensional Integrable Systems and Nambu-Poisson Geometry, J. Nonlinear Math. Phys. 8 (2001) 325-341.

[20] Guha P, Generalized Poisson Mechanics in D-Brane, Int. J. Mod. Phys. A. 17 (2002), 47594775 .

[21] Chatterjee R, Dynamical Symmetries and Nambu Mechanics, Lett. Math. Phys. 36 (1996), 117.

[22] Gonera C and Nutku Y, Superintegrable Calogero-type systems admit maximal number of Poisson structures, Phys. Lett. A 285 (2001), 301.

[23] Olshanetsky M A and Perelomov A M, Classical integrable finite-dimensional systems related to Lie algebras, Phys. Rep. 71, (1981) no. 5, 313-400.

[24] Guha P, Applications of Nambu Mechanics to systems of hydrodynamical type, Jour. of Math. Phys. 43 (2002), 4035-4040.

[25] Dubrovin B A and Novikov S P, Hydrodynamics of soliton lattices, Soviet Scientific Reviews, Section C: Mathematical Physics Reviews, 9, Part 4. Harwood Academic Publishers GmbH, Yverdon, 1993.

[26] Névir P and Blender R, A Nambu representation of incompressible hydrodynamics using helicity and enstrophy, J. Phys. A: Math. Gen. 26 (1993), L1189-L1193.

[27] Olver P, A nonlinear Hamiltonian structure for the Euler equations, J. Math. Anal. Appl. 89 (1982) no. 1, 233-250.

[28] Friedlander S and Vishik M M, Lax pair formulation for the Euler equation, Phys. Lett. A 148 (1990) no. 6-7, 313.

[29] Friedlander S and Vishik M M, An inverse scattering treatment for the flow of an ideal fluid in two dimensions, Nonlinearity 6 (1993) no. 2, 231.

[30] Li Y -C A Lax Pair for 2D Euler Equation, J. Math. Phys. 41 (2000), 728-758.

[31] Gibbon J D, A quaternionic structure in the three-dimensional Euler and ideal magnetohydrodynamics equations, Phys. D 166, (2002) no. 1-2, 17-28. 